\title{
Can We Explain the Memory Transfer between Generations by Mathematical Analysis of DNA Walk?
}

\author{
Hamidreza Namazi \\ 167 Division Ave Brooklyn, New York 11211, USA
}

*Corresponding Author: Hamidreza Namazi, 167 Division Ave Brooklyn, New York 11211, USA, E-mail;hrnneuromap@gmail.com

Received: June 21, 2017

Accepted: June 23, 2017
Published: June 24, 2017
Genetic memory can be discovered from two points of view. In biology, genetic memory is defined as memory which is present if the state of a biological system depends on its history in addition to present conditions. Genetic memory is recorded in the genetic material and stably inherited through cell division. On the other hand from psychological point of view, genetic memory is the memory which is present at birth when no sensory experience was imposed to the offspring. In fact scientists believe that this memory is incorporated into the genome over long spans of time.

In the 19th century, Lamarckian mechanism was the basis of genetic memory, where scientists considered genetic memory as a fusion of memory and heredity. Ribot stated that psychological and genetic memories are based upon a common mechanism, but psychological memory is interacted with consciousness [1]. Hering and Semon developed general theories of memory which brings the idea of the engram and concomitant processes of engraphy and ecphory into consideration [2].

Modern views bring up the idea that common experiences of a species become incorporated into its genetic code by a much indistinct tendency compared to Lamarckian process, which encode tautness to respond in certain ways to certain stimuli. There have been some studies that examined the transmission of stimulus-specific behavioral and structural adaptations in the nervous system from parents to their offspring. For instance, Stickrod et al. did experiments on rats and found out that in utero taste and odor aversion learning in mother affects the offspring's preference and avoidance of flavors and odors [3].In another study, Todrank et al. figure out that the mouse maternal diet with acetophenone at various stages of gestation increases M71 glomerular area and preference for acetophenone in adolescent offspring [4]. Carone et al. did similar investigation on paternal diet which showed the marked effects on the metabolic physiology of offspring conceived after the father's diet had been manipulated [5]. Some studies found out that the exposure to the antiandrogenic endocrine disruptor vinclozolin during embryonic gonadal sex determination affects fertility and behaviour in at least four subsequent generations. In fact, this effect is associated with epigenetic changes in the sperm of descendant male offspring [6-8]. Dietz et al. found an inheritance of depression-like behaviour in the forced swim test using IVF with sperm from socially defeated fathers. They indicated that paternal experience can affect the behaviour in offspring even if the offspring have not been conceived at the time of paternal trauma [9]. In another interesting study, Vassoler et al. investigated the epigenetic inheritance of a cocaine-resistance phenotype from father to offspring [10].In a recent outstanding work done by Dias and Ressler [11], mice trained to fear a specific smell. The results of their study showed that offspring were extremely sensitive and fearful of the same smell, even though they had never encountered it, nor been trained to fear it. They stated "There is absolutely no doubt that what happens to the sperm and egg will affect subsequent generations."They believed that the stimulus makes changes in DNA methylation, which occurs at relevant genetic loci. In fact, the results of all these studies emphasize that transgenerational epigenetic inheritance does occur in mammals. 
One useful approach to study the scaling properties of many biological time series and patterns is to apply methods derived from the concept of self-similar (fractal) processes. A fractal is a natural phenomenon or a mathematical set that exhibits a repeating pattern that displays at every scale [12]. The class of regular fractals includes many familiar simple objects, such as line intervals, solid squares, and solid cubes, and many irregular objects. The scaling rules are characterized by "scaling exponents" (dimension). "Simple" regular fractals have integer scaling dimensions [13]. Complex self-similar objects have noninteger dimension. Fractal theory has been used widely in biology and medicine for various cases such as DNA [14-15], eye movement [16], EEG signal [17-19], bone structure [20], respiration signal [21], heart rate [22] and human stride time series [23]. Two main features, which are discussed in case of analysis of fractal time series, are fractal dimension and the Hurst exponent.

In [24] we showed that in general the gradual reduction in the human memory during time can be seen in the Hurst exponent plots of the EEG signals [25] where the values of the Hurst exponents get closer to $\mathrm{H}=0.5$ as time goes on. On the other hand, the effect of a strong stimulus (bigger than threshold value) is mapped accordingly on the EEG's Hurst exponent plot by a sudden jump which deviates the spectra from $\mathrm{H}=0.5$. If a bigger stimulus is applied on the subject, accordingly bigger fluctuation can be seen the Hurst exponent plot. Consider the case that no stimulus applied to the human, so, the Hurst exponent plot gradually get closer to $\mathrm{H}=0.5$ which stands for chaotic state, In this condition $(\mathrm{H}=0.5)$ the EEG signal will be completely random. So, the stimuli are crucial to the survival of organisms and often manifests as alterations in the structure and function of the nervous system [26].

Based on the idea in the last mentioned paper, when we worked on diagnosis of skin cancer by analysing the Hurst exponent and fractal dimension plots [27], we saw small fluctuations in the Hurst exponent plots of DNA walks for different subjects (healthy or patient). In that paper, we hypothesized that this fluctuations are related to the memory of system. In fact, in analysing the DNA walk, our system is human genome. So, the fluctuations in the Hurst exponent plot should be related to the genetic memory.
In this paper using the observed phenomena in our latest research, we propose to study the DNA walk of father and offspring. In order to do this, we should extract the DNA sequence of gens in case of different subjects (father which was subjected to odor fear condition, and the offspring who didn't trained against any stimulus), and then using a popular method we generate a planar trajectory of DNA sequences (DNA walk). After that in order to study the effect of inherent transfer of memory from father to offspring, we will study the Hurst exponent variations for father and offspring in case of different samples. Based on our hypothesis we should see the effect of applied stimulus in the Hurst exponent plots of both father and offspring. In general it can be said that our hypothesis is based on the concept of the Hurst exponent which says "the Hurst exponent is an indicator of the long term memory of the process." So, we must be able to explain the genetic memory by Hurst exponent analysis.

For further comparison and accordingly verification, we will use the Fractal dimension as another indicator in order to compare the DNA walk of father and offspring. Bringing the Fractal dimension into the account is also based on our latest investigation, which says that application of external stimulus makes the DNA walk less complicated [28].

\section{REFERENCES}

[1] Louis D. Matzel (2002). "Learning Mutants". In Harold E. Pashler. Steven's Handbook of Experimental Psychology. John Wiley and Sons. p. 201. ISBN 0-471-65016-1.

[2] Timothy L. Strickler (1978). Functional Osteology and Myology of the Shoulder in the Chiroptera. Karger Publishers. p. 325. ISBN 38055-2645-8.

[3] Stickrod, G., Kimble, D.P. \& Smotherman, W.P. In utero taste/odor aversion conditioning in the rat. Physiol. Behav. 28, 5-7 (1982).

[4] Todrank, J., Heth, G. \& Restrepo, D. Effects of in utero odorant exposure on neuroanatomical development of the olfactory bulb and odour preferences. Proc. Biol. Sci. 278, 1949-1955 (2011).

[5] Carone, B.R. et al. Paternally induced transgenerational environmental reprogramming of metabolic gene expression in mammals. Cell 143, 1084-1096 (2010).

[6] Anway, M.D. Epigenetic transgenerational actions of endocrine disruptors and male fertility. Science 308, 1466-1469 (2005). 
[7] Crews, D. et al. Transgenerational epigenetic imprints on mate preference. Proc. Natl. Acad. Sci. USA 104, 5942-5946 (2007).

[8] Guerrero-Bosagna, C., Settles, M., Lucker, B. \& Skinner, M.K. Epigenetic transgenerational actions of vinclozolin on promoter regions of the sperm epigenome. PLoS ONE 5, e13100 (2010).

[9] Dietz, D.M. et al. Paternal transmission of stress-induced pathologies. Biol. Psychiatry 70, 408-414 (2011).

[10] Vassoler, F.M., White, S.L., Schmidt, H.D., Sadri-Vakili, G. \& Pierce, R.C. Epigenetic inheritance of a cocaine-resistance phenotype. Nat. Neurosci. 16, 42-47 (2012).

[11] Dias, Brian G., Ressler, Kerry J (2013). "Parental olfactory experience influences behavior and neural structure in subsequent generations". Nature Neuroscience 17 (1): 8996. doi:10.1038/nn.3594

[12] Hamidreza Namazi, Can We Correlate the Spider's Brain Activity to it Spinning Web Activity?. ARC Journal of Neuroscience. 2017; 2(1):17-18. doi:dx.doi.org/10.20431/2456057X.0201005.

[13] Namazi H, Akrami A, Haghighi R, Delaviz A, Kulish V.V. Analysis of the Influence of Element's Entropy on the Bulk Metallic Glass (BMG) Entropy, Complexity and Strength. Metallurgical and Materials Transactions A.DOI: 10.1007/s11661-016-3870-3 (2016).

[14] Namazi, H. \& Kiminezhadmalaie, M. Diagnosis of Lung Cancer by Fractal Analysis of Damaged DNA. Comput. Math. Methods Med. 2015; 1-13 (2015).

[15] Namazi, H. et al. The fractal based analysis of human face and DNA variations during aging. Biosci. Trends. 10, doi: 10.5582/bst.2016.01182 (2016).

[16] Namazi, H. Kulish, V. V. \& Akrami, A. The analysis of the influence of fractal structure of stimuli on fractal dynamics in fixational eye movements and EEG signal. Sci. Rep. 6, 26639; DOI: 10.1038/srep26639 (2016).

[17] Namazi, et al. A signal processing based analysis and prediction of seizure onset in patients with epilepsy. Oncotarget 7, 342-350; DOI: 10.18632/oncotarget.6341 (2016).

[18] Namazi, H., Akrami, A., Nazeri, S. \& Kulish, V. V. Analysis of the Influence of Complexity and Entropy of Odorant on Fractal Dynamics and Entropy of EEG Signal. BioMed. Res. Int. 2016, 5469587, 1-5; DOI:10.1155/2016/5469587 (2016).

[19] Namazi, H. et al. Analysis of the influence of memory content of auditory stimuli on the memory content of EEG signal. Oncotarget.7(35):56120-56128. doi: 10.18632/oncotarget.11234 (2016).

[20] Kyung-Hoe, Huh. et al. Fractal analysis of mandibular trabecular bone: optimal tile sizes for the tile counting method. Imaging Sci. Dent. 41, 71-78 (2011).

[21] Namazi, H., Akrami, A. \& Kulish, V. V. The Analysis of the Influence of Odorant's Complexity on Fractal Dynamics of Human Respiration. Sci. Rep. 6, 26948; DOI: 10.1038/srep26948 (2016).

[22] Namazi, H. and Kulish, V. V. Fractal Based Analysis of the Influence of Odorants on Heart Activity. Sci. Rep. 6, 38555; doi: 10.1038/srep38555 (2016).

[23] Namazi, H. \& Kulish, V. V. Mathematicalbased modeling and prediction of the effect of external stimuli on human gait. Int. J. Numer. Method Biomed. Eng. 32; DOI: 10.1002/cnm.2805 (2016).

[24] Hamidreza Namazi and Vladimir V. Kulish, "Fractional Diffusion Based Modelling and Prediction of Human Brain Response to External Stimuli," Computational and Mathematical Methods in Medicine, 2015, doi:10.1155/2015/148534 (2015).

[25] Namazi, H. \& Kulish V. V. Mathematical Modeling of Human Brain Neuronal Activity in the Absence of External Stimuli. Journal of Medical Imaging and Health Informatics 2(4):400-407 (2012).

[26] Namazi H, Kulish VV. A mathematical based calculation of a myelinated segment in axons. ComputBiol Med. 2013 Jul; 43(6):693-8. doi: 10.1016/j.compbiomed.2013.03.005.

[27] Hamidreza Namazi, Vladimir V. Kulish, Fatemeh Delaviz, Ali Delaviz. Diagnosis of skin cancer by correlation and complexity analyses of damaged DNA. Oncotarget, Advance Publications 2015. DOI: 10.18632/oncotarget.6003

[28] Namazi, H. et al. Mathematical Modelling and Prediction of the Effect of Chemotherapy on Cancer Cells. Sci. Rep. 5, 13583; doi: 10.1038/srep13583 (2015).

Citation: Hamidreza Namazi, Can We Explain the Memory Transfer between Generations by Mathematical Analysis of DNA Walk?. ARC Journal of Neuroscience. 2017; 2(2):1-3. doi:dx.doi.org/10.20431/2456057X.0202001.

Copyright: (C) 2017 Authors. This is an open-access article distributed under the terms of the Creative Commons Attribution License, which permits unrestricted use, distribution, and reproduction in any medium, provided the original author and source are credited. 